ULB-TH/96/14

hep-ph/9608266

\title{
Stimulated Neutrino Conversion and Bounds on Neutrino Magnetic Moments
}

\author{
J.-M. Frère \\ Université Libre de Bruxelles \\ R.B. Nevzorov, M.I. Vysotsky \\ ITEP, Moscow 117259, Russia
}

\begin{abstract}
Recent experiment proposed to observe induced radiative neutrino transitions are confronted to existing bounds on neutrino magnetic moments from earthbased experiments. These are found to exclude any observation by several orders of magnitude, unless the magnetic moments are assumed to be strongly momentum dependent. This possibility is discussed in some generality, and we find that nontrivial dependence of the neutrino form factor may indeed occur, leading to quite unexpected effects, although this is insufficient by orders of magnitude to justify the experiments.
\end{abstract}

\footnotetext{
${ }^{1}$ postal address: Physique Theorique CP 225; U.L.B. Boulevard du Triomphe; B-1050 Bruxelles, Belgium; email: frere@ulb.ac.be
} 
The following method of search for neutrino magnetic transitions was recently proposed in [1]: neutrinos pass a resonant cavity full of low-energy photons which induce the neutrino conversion: $\nu_{1} \gamma \rightarrow \nu_{2}$. While neutrino decay in vacuum is strongly suppressed by lack of phase space if the mass difference between $\nu_{1}$ and $\nu_{2}$ is small $\left(\left(\Gamma \sim(\delta m)^{3}\right)\right)$ in the case of stimulated conversion the energy is provided by the electromagnetic field, and the $\delta m$ suppression disappears. This fact makes a suggested way to look for the neutrino transitional magnetic moments very attractive. In paper [四] possible experiments with the electron neutrinos from a reactor or the Sun were discussed. The same idea was applied in paper [2] to accelerator-produced $\nu_{\mu}$ 's. The scheme of the experiment is obvious: neutrinos on the way to a standard detector pass through a high-quality resonant cavity; a variation in the detected neutrino fluxes associated to switching on and off the cavity is interpreted as the signal of a radiative transition. According to the estimates given [2], the suggested experiments could hope to detect transition neutrino magnetic moments $\mu$ larger than:

$$
\mu \geq 4 \cdot 10^{-4} \mu_{B}
$$

where $\mu_{B}$ is Bohr magneton.

Our purpose here is to study the compatibility of such large transition magnetic moments with existing bounds from earth-based experiments. A positive signal of stimulated transition could be observed in three different situations: (in what follows, we only mention neutrinos, parallel situations obviously happen with their antiparticles, or rather, their CP conjugates):

- apparition : an inactive (sterile) neutrino present in the beam is converted inside the cavity into an active one. The initial neutrino can be either a right-handed component of one of the known neutrinos, or a new, this far undetected particle altogether, while the neutrino emerging from the cavity is one of the known, (mostly) left-handed types $\left(\nu_{e}, \nu_{\mu}, \nu_{\tau}\right.$ or there antiparticles)

- disparition : an active neutrino disappears into an inactive species, either a new state, or the right-handed component of a (Dirac) known neutrino

- conversion : a known neutrino is converted into another species, itself observable, for instance a left-handed $\nu_{\mu}$ absorbs a $\gamma$ and turns into a righthanded (active) $\bar{\nu}_{e}$; the beam then appears depleted in one species, and enriched in another. 
What is common to all theses possibilities is that they always involve at least one transition moment to or from one of the 3 standard, relatively well-known neutrinos.

The bounds on neutrino magnetic moments are usually quoted in the litterature in terms of "diagonal" moments rather than transition moments. They are 3]:

$$
\mu_{\nu_{\mu}} \leq 10^{-9} \mu_{B}, \mu_{\nu_{e}} \leq 10^{-10} \mu_{B}
$$

The bound on the tau neutrino magnetic moment is stronger than (1) as well [3]:

$$
\mu_{\nu_{\tau}} \leq 5.4 \cdot 10^{-7} \mu_{B}
$$

We want to point out that the above bounds are in fact more general and do apply as well to transition magnetic moments, provided phase space is sufficient in the experiment considered. For this, it is easiest to reason in terms of helicity amplitudes, a natural approach for light, energetic neutrinos. Interference between helicity amplitudes only occurs through the neutrino masses, and can thus be safely neglected for most purposes.

These bounds above are deduced from the absence of any observed addition to the predicted standard model neutrino-electron scattering. While standard model weak contributions, mediated by the $\mathrm{Z}$ and $\mathrm{W}$ direct couplings are diagonal in the helicity representation (LL and RR transitions), as expected from a regular gauge interaction, an hypothetical magnetic coupling to the photon, being a tensor interaction, involves a LR transition. For this reason, the neutrino magnetic moment contribution never interferes with the standard contribution, and the cross sections thus add trivially. As a result, the respective natures of the initial and final neutrino are irrelevant, provided the mass of the final neutrino does not suppress the reaction. Thus, the resulting cross section (and the resulting bound) is the same, whether the final (undetected) neutrino is identical to (diagonal magnetic moment) or different from the first (transition moment). The bounds (2), (3) are applicable to any kind of a transitional magnetic moment, the only condition being the smallness of the mass difference between the two neutrino species, compared to the center of mass energy available in the process, which is about $100 \mathrm{MeV}$ for $\nu_{\tau}$ and several $\mathrm{MeV}$ for $\nu_{e}$ and $\nu_{\mu}$.

The comparison of (11), (2), (3) then seems to settle the issue.

A potential loophole however exits. The bounds (2), (3) were indeed 
obtained for virtual photons, with typical $q^{2}$ varying from $\sim(100 \mathrm{MeV})^{2}$ for $\nu_{\tau} e$-scattering and $\sim(1 \mathrm{MeV})^{2}$ for $\bar{\nu}_{e} e$ scattering, while radiative decays of neutrinos deals with real photons and absorption of a photon from the resonance cavity involves $q^{2} \sim\left(10^{-6} \mathrm{eV}\right)^{2}$. So we should, as devil advocates, consider the possibility that neutrino magnetic form factors build up with diminishing $\left|q^{2}\right|$, in which case even a strong upper bound at large $q^{2}$ (2), (3) could coexist with a considerably larger $\mu$ at small $q^{2}$, restoring hope for a cavity experiment. Let us look more closely at this possibility.

The neutrino magnetic moment in generalizations of the standard model is usually generated through a triangle diagram involving virtual charged particles. The dependence on $q^{2}$ is determined by the mass of the heaviest charged particle in the loop (usually it is a new scalar or vector boson) and for $q^{2}<M^{2}$ has the following form:

$$
\mu\left(q^{2}\right)=\mu(0)\left[1+a \frac{q^{2}}{M^{2}}\right] .
$$

Here $a$ is a model-dependent number of order 1 , while we know that $M$ has to be larger than $60 \mathrm{GeV}$ from the absence of new charged particles at LEP 1.5. It is thus clear from (4) that $\mu\left((100 \mathrm{MeV})^{2}\right)=\mu(0)$ with very high accuracy. Of course, renormalisability of the theory tells us that the formfactor has ultimately to decrease with $q^{2}$ for asymptotic $q^{2}$, but this only really takes place for $q^{2}>M^{2}$; and as there are no charged bosons lighter than $60 \mathrm{GeV}$ this cannot help us.

At this point, we might again give up, but devil's advocates tend to perseverate, and another possibility exists. The problem in generating the magnetic moment through charged particles loops, is that the masses of the new intermediaries have to be very large, as we know form LEP limits. The situation is different with neutral intermediaries, but the counterpart is that their coupling to photons can only be through an induced magnetic moment. We will consider as an example the situation where the transition magnetic moment between $\nu_{1}$ and $\nu_{2}$ is induced through a loop containing the new light fermion $N$, and a light scalar $\varphi$; the light fermion is assumed to have a magnetic moment $\mu_{N}$, possibly induced by heavy particles, and, basing ourselves on the previous case, we will consider $\mu_{N}$ to be constant in most of the integration range (as we will see, the infrared part of the integration is the relevant one). It is quite clear that both $N$ and $\varphi$ should be $\mathrm{SU}(2)$ singlets to avoid conflict with the measured $Z$-boson invisible width. For 
the simplicity of the argument, we will for now consider only one neutrino and compute the induced diagonal magnetic moment; the calculation below carries over essentially unchanged for transition moments.

Let us take the standard neutrino $\nu_{1}$ to be a Dirac particle coupling with $N$ through the Yukawa interaction $f \bar{\nu} N \varphi$, to the new scalar particle. Let us note in passing that this interaction, if $\nu_{1}$ is to be observable, involves one element of an SU(2) doublet, with singlets. Such coupling is only possible as a consequence of the standard model $\mathrm{SU}(2)$ breaking acting on an induced vertex; we might therefore expect that it is somewhat suppressed, but this is not essential.

It is convenient to present the neutrino magnetic formfactor in the following form:

$$
\mu_{\nu}=\frac{f^{2} \mu_{N}}{16 \pi^{2}} I\left(m_{N}, m_{\varphi}, m_{\nu}, q^{2}\right)
$$

where $I$ is a dimensionless function. For momentum transfer $q^{2}$ larger than the masses of all three particles we get a constant result $I=-1 / 2$ ?, instead of the anticipated decrease of the form factor (this is no real surprise, as an implicit cut-off is present, and the decrease is only expected for momenta larger than the masses of the charged particles responsible for the magnetic moment of the fermion $N$ ). In most cases (except for fine tuning, see below) at $q^{2}=0 I$ is of the order of unity, and the bounds on $\mu_{\nu}$ from the $\nu e$-scattering experiments stay applicable to neutrino behavior at small $q^{2}$, $\mu_{\nu}\left(q^{2}=(100 \mathrm{MeV})^{2}\right) \approx \mu_{\nu}\left(q^{2}=0\right)$. However, some interesting exceptions take place when the Feynman integral at small $q^{2}$ is infrared divergent. For example, let us suppose that the masses of the external neutrino and of the new spinor $N$ are almost degenerate, while the mass of the scalar $\varphi$ almost vanishes (like in a Goldstone boson situation):

$$
m_{\nu} \approx m_{N} \equiv m, m_{\varphi} \ll m_{\nu}-m_{N} .
$$

Performing the integration we get:

$$
I=-\frac{1}{2}+\frac{4 m^{2}}{-q^{2}}\left[\ln \left(\frac{m^{2}}{m_{N}^{2}-m_{\nu}^{2}}\right)-\frac{3}{4}\right] \frac{2}{\sqrt{1+\frac{4 m^{2}}{-q^{2}}}} \ln \left(\frac{\sqrt{1+\frac{4 m^{2}}{-q^{2}}}+1}{\sqrt{1+\frac{4 m^{2}}{-q^{2}}}-1}\right)+\delta\left(q^{2}\right)
$$

\footnotetext{
${ }^{2}$ this result is obtained by symmetrical integration in 4 dimensions. Other regularization methods can differ by a constant term (e. g. dimensional regularization). This however is typical of effective theories and does not affect our discussion
} 


$$
\begin{gathered}
\delta\left(q^{2}\right)=\frac{4 m^{2}}{-q^{2}}\left[\frac{1}{\sqrt{1+\frac{4 m^{2}}{-q^{2}}}} \ln \left(\frac{\sqrt{1+\frac{4 m^{2}}{-q^{2}}}+1}{\sqrt{1+\frac{4 m^{2}}{-q^{2}}}-1}\right) \ln \left(4+\frac{-q^{2}}{m^{2}}\right)+\right. \\
\left.+\frac{2}{\sqrt{1+\frac{4 m^{2}}{-q^{2}}}}\left(F\left(\frac{-1-\sqrt{1+\frac{4 m^{2}}{-q^{2}}}}{2 \sqrt{1+\frac{4 m^{2}}{-q^{2}}}}\right)-F\left(\frac{1-\sqrt{1+\frac{4 m^{2}}{-q^{2}}}}{2 \sqrt{1+\frac{4 m^{2}}{-q^{2}}}}\right)\right)\right] \\
F(\xi)=\int_{0}^{\xi} \frac{\ln (1+x)}{x} d x \\
I=-\frac{1}{2},\left|q^{2}\right| \gg m^{2} \\
I=4 \ln \left(\frac{m}{2\left(m_{N}-m_{\nu}\right)}\right)-\frac{7}{2},\left|q^{2}\right| \lesssim m^{2}
\end{gathered}
$$

In this case, the magnetic formfactor of neutrino at $q^{2}=0$ can thus in principle be much larger than at large momentum transfer.

Let us note that while the constant term in $I$ may depend on the particular mehanism of $\mu_{N}$ generation at high energies, the logarithmic term in $I$ originates in the infrared and is universal.

From (10) and (5), and the requirement to stay in a regime where we can still trust perturbation theory, we can deduce the largest possible value for the neutrino magnetic moment at $q^{2}=0$ : (further tuning would at least force the resummation of the leading terms)

$$
\mu_{\nu}^{\max }\left(q^{2}=0\right)=\mu_{N}
$$

Even accepting such a strong tuning (and generalizing straightforwardly to transition moments of nearly degenerate neutrinos) in a rather ad-hoc scheme does not allow any induced radiative transition to be observed in the suggested cavity experiment, as strict bounds still apply to $\mu_{N}$.

We review them briefly, starting from earth-based experiments and moving to astrophysical ones.

The bound from ref. [4] on the $\nu_{\tau}$ magnetic moment generalizes straightforwardly to any light neutral fermion $\mathrm{N}$ which can be produced in the reaction $e^{+} e^{-} \rightarrow N \bar{N} \gamma$ :

$$
\mu_{N}<4 \cdot 10^{-6} \mu_{B}
$$


It is interesting to note that numerically close bound can be obtained from measured at LEP invisible width of Z-boson. Here the line of reasoning goes as follows. If neutral fermion is magnetically coupled to photon, it should couples to Z-boson as well with strength damped by at most square of electroweak mixing angle sine. In this way invisible $\mathrm{Z}$ width get additional contribution and experimental bound on the last leads to bound on magnetic moment.

While this might still allow for a rather generous low momentum transfer (transition) magnetic moments through the above-proposed mechanism,this limit is comfortably stronger than (11). Stronger bounds come from astrophysical data. The bound from the white dwarf cooling [5] $\mu_{N}<3 \cdot 10^{-11} \mu_{B}$ is much stronger. Let us remind that this white dwarf bound is valid for masses of $N$ lower than $\omega_{p} / 2 \approx 20 \mathrm{KeV}$; since heavier particles can not be produced in plasmon decay. As we have seen, the low-momentum transfer enhancement only occurs for nearly-degenerate particles. So for a $\nu_{e}$ magnetic moment generated through the proposed mechanism the white dwarf bound is applicable (as $m_{N} \approx m_{\nu_{e}}<4 \mathrm{eV}$ ); while for $\nu_{\mu}$ and $\nu_{\tau}$ magnetic moments values up to the bound (12) are still allowed if they (and hence $N$ ) happen to be heavier than $20 \mathrm{KeV}$.

In conclusion we demonstrate that a nontrivial $q^{2}$ dependence, resulting in a strong low-momentum enhancement of a neutrino magnetic form factor is possible: the bounds from $\nu$-scattering experiments at $q^{2} \sim(1 \mathrm{MeV})^{2} \div$ $(100 \mathrm{MeV})^{2}$ are not directly applicable to the neutrino magnetic moment which governs the neutrino behavior in a resonant cavity, in a constant magnetic field, or even to radiative decays.

The construction leading to such enhancement (and presented more as a devil's advocate's argument) involves as a necessary ingredient a light neutral fermion $N$ with a nonzero magnetic moment, and the enhancement has been shown to apply if a fine tuning of the masses leads to a nearly divergent behaviour of the relevent loop integral in the infrared.

Even this possible enhancement cannot save the hope of observing induced radiative transitions using a resonant cavity, due to the strong LEP limits on the magnetic moment of any light particle, including the hypothetical $N$. Despite our efforts as devil's advocates, the beautiful idea of serching for stimulated neutrino conversion in a high-quality resonant cavity [1], [2] stays less sensitive than the experimental bounds (21), (3), (11), (12) ( by at least two orders of magnitude in the magnetic moments, that is four orders 
of magnitude in the number of events).

As a complement to the present study, let us note recent proposal [6] to look for neutrino magnetic moment in reaction $\nu e \rightarrow \nu e \gamma$, where the interesting region of small intermediate photon virtuality can be investigated.

We thank M.C.Gonzalez-Garcia for a discussion of experimental bounds on neutral fermion magnetic moments, and JMF acknowledges at this occasion the support of the european network Flavourdynamics (chrx-ct93 -0132).

The investigations of J.-M.Frère, R.Nevzorov and M.Vysotsky were supported by INTAS grant 94-2352; those of R.Nevzorov and M.Vysotsky were supported by the grant RFBR-96-02-18010 as well.

\section{References}

[1] S.Matsuki and K.Yamamoto, Phys. Lett. B289 (1992) 194.

[2] M.C.Gonzalez-Garcia, F.Vannucci and J.Castromonte, Phys. Lett. B373 (1996) 153.

[3] Phys. Rev. D50 (1994) 1173, Review of Particle Properties.

[4] H.Grotch and R.W.Robinett, Zeit. fur Physik C 39 (1988) 553.

[5] S.I.Blinnikov, preprint ITEP-19 (1988);

S.I.Blinnikov, V.S.Imshennik and D.K.Nadyozhin, Sov. Sci. Rev. E6, 266 (1988).

[6] J.Bernabeu, S.M.Bilenky, F.J.Botella and J.Segura, Nucl.Phys. B246 (1994) 434. 